\title{
V. 3, N. (1), Jan./Abr. 2018
}

UNIVERSIDADE FEDERAL DE ALAGOAS

\author{
Maria Valéria Costa Correia \\ Reitora
}

José Vieira da Cruz

Vice-Reitor

\section{FACULDADE DE MEDICINA}

Francisco José Passos Soares

Diretor

Mércia Lamenha Medeiros

Vice-Diretora

NÚCLEO DE SAÚDE PÚBLICA

\section{EQUIPE EDITORIAL}

Divanise Suruagy Correia Faculdade de Medicina - UFAL Editora

Divanise Suruagy Correia Universidade Federal de Alagoas

Rosana Quintella B. Vilela Universidade Federal de Alagoas

Claudia Santos Martiniano Universidade Estadual da Paraíba

Vilma Leyton

Universidade de São Paulo

Eulália Maria Chaves Maia Universidade Federal do Rio Grande do Norte

Geraldo Marcelo Cunha Escola Nacional de Saúde Pública Sergio Arouca

Margarete Pereira Cavalcante Universidade Federal de Alagoas

Conselho Editorial
Vilma Leyton

Universidade de São Paulo

Eulália Maria Chaves Maia

Universidade Federal do Rio Grande do Norte

Geraldo Marcelo Cunha

Escola Nacional de Saúde Pública Sergio Arouca

Daniel Antunes Freitas

Universidade Estadual de Montes Claros

Waneska Alexandra Alves

Universidade Federal de Juiz de Fora

Rosana Quintella B. Vilela

Universidade Federal de Alagoas

Comitê Consultivo

Divanise Suruagy Correia

Faculdade de Medicina - UFAL

$$
\text { Normalização }
$$

Paulo Rozendo da Paixão

Universidade Federal de Alagoas

Adenize Ribeiro da Silva

Universidade Federal de Alagoas

Equipe Técnica

Almira Alves Santos

Universidade Estadual de Ciências da Saúde de Alagoas

Angela Maria Moreira Canuto Universidade Federal de Alagoas

Claudia Santos Martiniano

Universidade Estadual da Paraíba

Daniel Antunes Freitas

Universidade Estadual de Montes Claros

Divanise Suruagy Correia

Universidade Federal de Alagoas

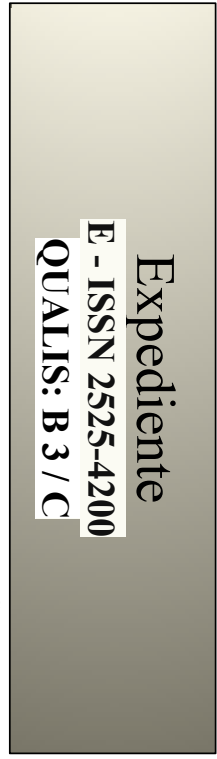


Eulália Maria Chaves Maia Universidade Federal do Rio Grande do Norte

Isis Suruagy Correia Moura Universidade Federal de Pernambuco

Jorge Luís de Souza Riscado Universidade Federal de Alagoas

José Rodrigues Rocha Junior Centro Universitário Tiradentes

Maria das Graças M. M. Taveira Universidade Federal de Alagoas

Margarete Pereira Cavalcante Universidade Federal de Alagoas
Rosana Quintella B. Vilela Universidade Federal de Alagoas

Sandra Lopes Cavalcanti Universidade Federal de Alagoas

Thatiana Regina Fávaro Universidade Federal de Alagoas

Valfrido Leão de Melo Neto Universidade Federal de Alagoas

Waneska Alexandra Alves Universidade Federal de Juiz de Fora Equipe de Avaliadores Gerais

AVALIADORES DO V. 3, N. 1, JAN./ABR. 2018

Adenize Ribeiro da Silva Claudia Santos Martiniano Cristina Camelo de Azevedo

Divanise Suruagy Correia Maria das Graças M. M. Taveira

Sandra Lopes Cavalcanti

Waneska Alexandra Alves

Divanise Suruagy Correia

Paulo Rozendo da Paixão

Editoração Eletrônica

Paulo Rozendo da Paixão

Capa

Tel.: +55 82 99964-0209

E-mail: revistaportalsaudeesociedade@gmail.com

Contatos 